\title{
EDITORIAL
}

\section{Polymer Journal for next generation of polymers and related materials}

Polymer Journal (2015) 47, 1; doi:10.1038/pj.2014.117

$\mathrm{I}$ wish you a Happy New Year. In this year, Polymer Journal publishes volume 47 since the foundation in 1970. This year is the sixth year of the collaboration of the Society of Polymer Science, Japan (SPSJ) with the Nature Publishing Group (NPG). In the year of 2014, ${ }^{1}$ we published a total of 123 papers including 80 Original Articles, 3 Rapid Communications, 13 Notes, 23 Focus Reviews and 4 Invited Reviews. These papers present important insights in polymer science for the future. In particular, Focus Reviews that started in 2012 are written by many young talented polymer scientists. In addition to regular issues of 2014, we published the special issue on Biorelated Polymers and Materials $^{2}$ (Guest Editor: Professor Serizawa). This area is becoming very important because of the demand for the development of artificial organs and drug delivery systems with related fields. Polymer materials are able to significantly contribute to this area. This special issue consisted of 11 excellent papers from China, Taiwan, Singapore, Thailand, Australia and Japan.

Our website www.nature.com/pj receives ten thousands of visits every month. The number of the visits is still increasing steadily. In addition to the visit to newly published paper, some of our classic papers are still downloaded. For example, a paper written by Dr D. A. Tomalia $^{3}$ is still downloaded. This paper was published in 1985 and it is still cited. The total number of citation of the paper is now more than 2100. It is our hope that many landmark papers will be published in Polymer Journal.

I also would like to introduce PJ Zeon Award that had started from 2005 (http://main.spsj.or.jp/c15/pjzaward/pjzjyuichiran-e.htm). This award is open to the first authors of papers published in Polymer Journal, who are under the age of 38 years at the time of submitting their paper. ${ }^{4}$ Each year the SPSJ selects up to three most outstanding papers. Until now, a total of 25 papers are awarded. I would like invite all possible authors to apply for this award. I would like to thank Zeon Corporation for the generous support.
In February 2015, we plan to publish a special issue on 'Fusion Materials'. This issue is based on the 'MEXT (The Ministry of Education, Culture, Sports, Science, and Technology)' project (https://www.fusion-materials.org/en/). 'Fusion materials' is a concept of new futuristic materials: sophisticated hybrid materials that are obtained through fuse of organic molecules and inorganic substances. About 25 excellent papers collected from the project members will be published in the issue. This is a first trial wherein one issue is covered by the results of a group project. I hope this issue also attracts great deal of attention as well as the regular issues and other special issues.

The impact factor, which is not the only measure for the quality of the journal, has increased from 1.133 in 2010 to 1.554 in 2014 (Journal Citation Reports). It is a good sign that Polymer Journal is becoming more visible.

Finally, I would like to thank the authors, readers, referees, associate editors and staffs of SPSJ and NPG for all of your continuous support and assistance.

Takashi Kato

Editor-in-Chief Polymer Journal

1 Kato, T. Polymer Journal: building on tradition for the future. Polym. J. 46, 1 (2014). 2 Serizawa, T. Special issue: biorelated polymers and materials. Polym. J. 46, 435 (2014).

3 Tomalia, D. A., Baker, H., Dewald, J., Hall, M., Kallos, G., Martin, S., Roeck, J., Ryder, J. \& Smith, P. A new class of polymers: starburst-dendritic macromolecules. Polym. J. 17, 117-132 (1985).

4 Kato, T. PJ ZEON Award for outstanding papers in Polymer Journal 2013. Polym. J. 46, 313-314 (2014). 